\title{
Can We Identify Emotion Over- regulation in Infancy? Associations with Avoidant Attachment, Dyadic Emotional Interaction and Temperament
}

\author{
Eva Costa Martins ${ }^{a, *}$, Isabel Soares ${ }^{b}$, Carla Martins ${ }^{c}$, \\ Susana Tereno ${ }^{\mathrm{d}, \mathrm{e}}$ and Ana Osório ${ }^{\mathrm{f}}$ \\ a Department of Psychology and Communication/ UNIDEP-CINEICC, Instituto \\ Superior da Maia, Maia, Portugal \\ ${ }^{\mathrm{b}}$ Department of Applied Psychology, School of Psychology, University of Minho, \\ Braga, Portugal \\ 'Department of Basic Psychology, School of Psychology, University of Minho, \\ Braga, Portugal \\ ${ }^{\mathrm{d}}$ Institut de Psychologie, Univ. Paris Descartes, Sorbonne Paris Cité, Paris, France \\ 'Laboratoire de Psychologie et Processus de Santé (LPPS-EA4057), Paris, France \\ ${ }^{\mathrm{f}}$ Neuropsychophysiology Lab, CIPsi, School of Psychology, University of Minho, \\ Braga, Portugal
}

Emotion over-regulation in infancy has seldom been the focus of empirical research. This study analysed the specificities of overregulation when compared with under-regulation (maladaptive) and adaptive regulation by testing its association with attachment, dyadic emotional interaction, and temperament. The sample consisted of 52 low-risk mother-infant dyads. During a home visit, dyadic emotional interaction was assessed in the daily routines and free play of 10-month-old infants. The infant's emotion regulation was assessed using the Shape Sorter Task, and a temperament questionnaire was completed by the mother. Attachment was assessed at 12 or 16 months using the Strange Situation. As hypothesized, (i) emotion overregulation (versus adaptive regulation) was predicted by a lower quality of dyadic emotional interaction and marginally by avoidant attachment; (ii) over-regulation (versus under-regulation) was predicted by avoidant attachment; and (iii) the predictive role of avoidant attachment was substantiated after controlling for another measure of

\footnotetext{
*Correspondence to: Eva Costa Martins, Department of Psychology and Communication/ UNIDEP-CINEICC, Instituto Superior da Maia, Av. Carlos Oliveira Campos, Castelo da Maia, 4475-690 Avioso S. Pedro, Portugal. E-mail: emartins@ismai.pt
} 
mother-infant interaction. Contrary to expectations, temperament did not distinguish between emotion regulation styles. The link between over-regulation and lower quality of mother-infant emotional interaction and avoidant attachment was demonstrated. There is empirical support to the claim that it is possible to identify emotion overregulation in infancy and that it is a maladaptive style of emotion regulation. Copyright (c) 2012 John Wiley \& Sons, Ltd.

Key words: emotion regulation style; emotion over-regulation; avoidant attachment; mother-infant emotional interaction; infant temperament

Generally speaking, emotion regulation can be defined as the intrinsic (e.g., physiological) and extrinsic (e.g., behavioural, social) processes associated with the activation of an emotion and its management over time (Cole, Martin, \& Dennis, 2004; Gross \& Thompson, 2007). These processes have an impact on the overall functioning of an individual, influencing the adaptive or maladaptive role of that emotional experience in a particular context (Cicchetti, Ganiban, \& Barnett, 1991; Cole et al., 2004; Gross \& Thompson, 2007). This topic of research has received increased attention in the last two decades, with numerous studies attesting to the importance of emotion regulation skills for adaptive functioning across developmental domains and psychopathology (Calkins, 2010; Dillon, Deveney, \& Pizzagalli, 2011).

Two opposite styles of maladaptive emotion regulation have been identified: under-regulation and over-regulation (Cassidy, 1994; Cole, Michel, \& Teti, 1994; Keenan, 2000). Both categories describe individuals who perseverate on using the same strategies to deal with emotion activation precluding the necessary emotional flexibility to respond to changes in environment and personal demands. Underregulation is characterized by the intensification of emotions in different contexts, including heightened negative emotionality and vigilance as well as overdependence on others for regulation (Cassidy, 1994; Keenan, 2000; Sroufe, 1996). Over-regulation is defined by a minimization of emotional expression leading to a constricted range of emotional experience associated with a lack of openness (e.g., less emotional expression) and regulatory behaviours that rely mostly on the self (e.g., self-soothing and object-oriented) (Cassidy, 1994; Main, 1990; Sroufe, 2000).

Research with adults has confirmed the maladaptive role of emotion overregulation by demonstrating an association between the suppression of distressing thoughts or emotions and psychopathology as well as negative social functioning (Aldao \& Nolen-Hoeksema, 2010; Srivastava, Tamir, McGonigal, John, \& Gross, 2009). Additionally, Maughan and Cicchetti (2002) identified emotion overregulation as a frequent dysregulated emotion pattern in maltreated children. Although there is growing awareness that emotion over-regulation is maladaptive, investigation focusing on this style of emotion regulation and its developmental trajectory in infancy is still scarce. Most research on emotion regulation continues to operationalize the maladaptive style of emotion regulation as the high expression of negative emotionality (i.e., under-regulation), while over-regulation remains mostly unaddressed (for a recent example; see Graziano, Keane, \& Calkins, 2010). The lack of research on emotion over-regulation may be because of assessment obstacles. Indeed, over-regulatory infants tend to use self-soothing and object-oriented strategies, which have been shown to be effective in inhibiting the expression of negative affect (Kopp, 1989). These strategies allow them to remain focused on the tasks at hand, appearing functional. Therefore, when studying infants, it is difficult to determine when the absence of negative and positive emotionality in connection with specific emotion regulation strategies is indicative 
of emotional dysregulation. Both in research and in everyday life, an infant or child that demonstrates over-regulation may not be easily identified as having emotion regulation difficulties. Bearing in mind that different strategies for emotion regulation seem to become organized into patterns or styles at the end of the first year of life (Diener, Mangelsdorf, McHale, \& Frosch, 2002; Kopp, 1989), we devised a measure aimed at capturing the patterning of emotion regulation skills that includes over-regulation, under-regulation and adaptive emotion regulation: Shape Sorter Task. The chosen interactional measure is both challenging and object-focused because this context is preferential for the assessment of emotion regulation in infancy in comparison with free play (Miller, McDonough, Rosenblum, \& Sameroff, 2002). By devising this measure that targets over-regulation in infancy, we aimed at contributing to the early identification of emotional over-regulation.

Most available research on emotion over-regulation comes from the attachment literature (Braungart-Rieker, Garwood, Powers, \& Wang, 2001; Crugnola, Tambelli, \& Spinelli, 2011; Diener et al., 2002; Koulomzin, Beebe, Anderson, Jaffe, Feldstein, \& Crown, 2002; NICHD Early Child Care Research Network, 2004; Volling, McElwain, Notaro, \& Herrera, 2002). This seems to be the case because the development of emotion regulation is embedded in the caregiving environment in general, and the attachment relationship in particular (Calkins \& Hill, 2007; Morris, Silk, Steinberg, Myers, \& Robinson, 2007; Sroufe, 2000). Nevertheless, the relations between over-regulation and other dimensions of the quality of mother-infant interaction or infant temperament have rarely been analysed (see Eisenberg, Hofer, \& Vaughan, 2007 for an exception). Given the importance of this topic and the limited empirical research available, this investigation focused on characterizing the style of emotion over-regulation in infancy through the analysis of its associations with three hypothesized variables implicated in its development: (i) avoidant attachment; (ii) quality of dyadic (mother-infant) emotional interaction; and (iii) infant temperament.

Attachment research indicates that a secure pattern will influence the development of an adaptive regulatory strategy, while insecure attachments will be linked to emotion dysregulation (Sroufe, 2000; Thompson, 2008). In the latter group, insecure resistant attachment has been typically associated with an under-regulated emotional style. In contrast, avoidant attachment might be associated with overregulation, as these infants are known to minimize their emotional expression, inhibit their emotion communication to the attachment figure and, therefore, rely significantly less on the parent for affect regulation (Cassidy, 1994; Main, 1990; Sroufe, 2000). Similarly, Meins et al. (2011) reported that avoidant children at 15 months were less prone to initiate joint attention behaviours with the mother, while the opposite was found with an experimenter. These results suggest a general mode of interaction (Meins et al., 2011) characterized by distancing from the caregiver regardless of whether the child is emotionally activated. This pattern seems to continue later in life, as Corriveau et al. (2009) discovered that 5-year-olds relied more on the answer provided by an experimenter than by their own mothers when asked to label or indicate the function of certain objects that were not familiar to them.

There is evidence to support the relationship between avoidant attachment and over-regulation in school-aged children and adults (Diamond \& Fagundes, 2008; Mikulincer \& Shaver, 2008; Sroufe, 2000), but data concerning infants and toddlers are not as straightforward. Although avoidant attachment to the mother has been related to some features of emotion over-regulation (Braungart \& Stifter, 1991; Crugnola et al., 2011; Koulomzin et al., 2002), there are other studies that indicate no association (Braungart-Rieker et al., 2001; NICHD Early Child Care Research Network, 2004; Volling et al., 2002). Another study by Diener and coworkers (2002) 
found a relationship within the father-child dyad but not with the mother. The findings available do not suggest a definitive influence of avoidant attachment on the development of over-regulation in infancy as attachment researchers initially hypothesized. Therefore, this remains an open research question. To explore this topic in this investigation, we analysed the following: (i) the implications of assessing emotion over-regulation and attachment in different contexts and (ii) the importance of attachment compared with other measures of infant-mother interaction for over-regulation.

First, we proposed that the theoretical link between avoidant attachment and over-regulation would be further supported if the infant's emotion regulation style was assessed in a context other than the Strange Situation Procedure (SSP; Ainsworth, Blehar, Waters, \& Wall, 1978). The SSP scoring system involves the analysis of many of the emotion regulation skills available to infants (e.g., increasing proximity with mother when distressed). An empirical overlap in the operationalization of attachment and emotion regulation could be argued to be on the basis of the associations found between the two (e.g., Crugnola et al., 2011). Therefore, finding a connection between attachment measured in a laboratory setting and emotion regulation assessed in the infant's home would provide evidence against this argument. As a result, in this research, we decided to observe emotion regulation in the infant's home. In addition, assessing emotion regulation in real-world contexts is an existing directive from emotion regulation researchers (Campos, Walle, Dahl, \& Main, 2011) seldom employed in infant research as most studies are laboratory based (Diener et al., 2002).

Second, we explored the importance of attachment compared with other measures of infant-mother interaction. Interestingly, some studies that have failed to find an association between emotion regulation and security of attachment in general, or avoidant attachment specifically, have uncovered a relationship with lower quality of mother-child interaction (Braungart-Rieker et al., 2001; NICHD Early Child Care Research Network, 2004; Volling et al., 2002). In this study, we aimed to explicitly assess the relative importance of avoidant attachment and the quality of motherchild interaction as predictors of over-regulation.

In addition to clarifying the role of avoidant attachment on over-regulation, we also analysed the contribution of the quality of infant-mother interaction and infant temperament. Mother-child interactions take many forms. They can range from play (which is not necessarily relevant for the development of the attachment bond) to situations in which the child is frightened (which are certainly relevant for the development of the attachment bond; see Goldberg, Grusec, \& Jenkins, 1999). As emotion over-regulation is a maladaptive style, it is expected to emerge from poorer parent-child interactions. One of the few studies outside of attachment literature that illustrates this link is Maughan and Cicchetti's (2002). They observed that maltreated 5-year-olds were more prone to develop dysregulated emotional patterns, one of which was the over-controlled/unresponsive type. Thus, it seems important to focus on both parent-child exchanges and infant attachment (Diener et al., 2002) to identify and understand over-regulation. Highlighting the contribution of both elements in a dyadic interaction, some researchers claim that an assessment of the quality of mother-infant interaction should comprise measures focused on the two-way emotional dialogue between caregiver and child (e.g., interactive contingency, Beebe, Jaffe, Markese, Buck, \& Chen, 2010; emotional availability, Biringen et al., 2005). This approach is supported by empirical studies showing a connection between mother-child affect exchanges and behavioural problems (Newland \& Crnic, 2011). Therefore, in this investigation, we have assessed two-way caregiverinfant interactions, focusing on their emotional exchanges that are defined in terms of dyadic emotional interaction. 
Temperamental differences, namely the infants' tendency to become emotionally aroused and affectively negative, have been found to directly and indirectly influence the quality of the emotion and behaviour regulatory skills and strategies developed by children (Calkins \& Hill, 2007). For example, a mother's perception of infant's difficult temperament measured at 3 and 9 months was related to variability in self-control in toddlerhood (Feldman, Greenbaum, \& Yirmiya, 1999). Looking specifically at overregulation, Eisenberg et al. (2007) hypothesized that some temperament characteristics are associated with emotion over-control, such as involuntary reactive control (behavioural inhibition in novel contexts and rigid over-control in the expression of emotion) and average or low effortful control. No other investigations have focused on this topic. Hence, further research is needed to clarify the associations between temperament and emotion over-regulation associated with emotional suppression. In our research, we investigated if over-regulated infants would be classified as easier babies, that is, less prone to cry and easier to soothe (Bates, Freeland, \& Lounsbury, 1979).

\section{AIMS AND HYPOTHESES}

There is a considerable gap in current knowledge regarding over-regulation of emotion in infancy. In this report, we examined the specificities of over-regulation by comparing it with both emotion under-regulation (maladaptive) and an adaptive emotion regulation style. Our main objective consisted of characterizing the overregulation of emotion by testing its relation to variables that may be implicated in its development, namely avoidant attachment, quality of mother-infant emotional interaction, and infant temperament. To accomplish this goal, we created a new assessment procedure-Shape Sorter Task-that allowed us to identify different styles of emotion regulation, including over-regulation, which is quite often absent from assessments of emotion regulation in infants.

We devised four hypotheses: (i) Avoidant attachment is associated with overregulation, when compared with adaptive emotion regulation or under-regulation. As has been previously suggested, avoidant attachment is linked to the development of over-regulation, secure attachment predicts adaptive emotion regulation, and ambivalent attachment predicts under-regulation (Sroufe, 2000). (ii) Lower quality of dyadic emotional interaction is associated with over-regulation when compared with adaptive emotion regulation. Dyadic emotion interaction would not, however, differentiate over-regulation from under-regulation as both are considered maladaptive styles and hence associated with lower quality of mother-child emotional interaction. (iii) Avoidant attachment predicts over-regulation beyond the quality of dyadic emotional interaction when compared with under-regulation and adaptive emotion regulation. This hypothesis is related to our intention to analyse the link between attachment and over-regulation, while controlling for the effect of dyadic emotional interaction (another index of the quality of mother-infant interaction). (iv) An easier temperament is associated with over-regulation when compared with other styles, as over-regulated infants are expected to exert high control over their emotional displays. Therefore, we expected that they would be regarded by their mothers as easier babies.

\section{METHOD}

\section{Participants}

This study was part of an ongoing longitudinal project, which has been following 52 low-risk, White, intact families (mother, father, and their infant). This paper focused 
only on the mother-infant dyads. Infants under the age of 10 months were recruited from nursery schools in Porto's metropolitan area (Portugal). Each family was contacted by the director of the nursery school who presented a letter with the research procedure and invited them to participate in the study. Families who showed interest were contacted by the research team by phone. At the time of the first assessment, mothers signed an informed consent. The infants (61\% male) were 10 months old $(M=306$ days, $S D=22.7)$. Mothers' socio-economic status was determined using Graffar's schedule (1956), and 48.1\% were classified as high $(n=25), 21.2 \%$ as middle-to-high $(n=11), 17.3 \%$ as middle $(n=9)$, and $13.5 \%$ as middle-to-low $(n=7)$.

\section{Procedure}

When the infants were 10 months old, a 40-min interaction between the mother and the infant in their home was video-recorded. In addition, mothers were asked to fill out a temperament questionnaire, a socio-demographic questionnaire, and an informed consent. During the first $20 \mathrm{~min}$, the mother was asked to go about her normal routine and in the following $10 \mathrm{~min}$ to play as she wished with the child. These first two assessments amounted to a total of $30 \mathrm{~min}$ of interaction and were used to score the quality of the dyadic emotional interaction. In the additional $10 \mathrm{~min}$, mothers were asked to play with a shape sorter provided by the researcher. This procedure was used to score emotion regulation. At 12 or 16 months, the dyad came to the laboratory for the SSP. Because these results were part of a larger study with the mother and father, assessments were counterbalanced. There was no association between attachment distribution to the mother and whether the infant was assessed at 12 or16 months, Cramer's V =.29, $p=.116$.

\section{Measures}

\section{Socio-demographic variables}

A socio-demographic questionnaire was used featuring questions related to sociodemographic variables: level of education, occupation, living arrangements, mother's and child's birth date, and infant's sex. Socio-economic status was determined using Graffar's schedule (1956), and each family was assigned to one of five categories from $1=$ High to $5=$ Low .

\section{Dyadic emotional interaction}

Dyadic emotional interaction refers to the capacity of both elements of the dyadmother and infant- to communicate emotionally and become affectively attuned to each other. This was assessed using three scales of the third edition of the Emotional Availability Scales (Biringen, Robinson, \& Emde, 1998). A composite variable combining maternal sensitivity and two child engagement scales-child responsiveness and child involvement-was created, Cronbach's $\alpha=.95$, by summing the individual scale scores. This new composite was named dyadic emotional interaction. Sensitivity (range 1-9) assesses the parent's capacity to read the child's emotional cues and to be emotionally responsive to the child. Child Responsiveness (range 1-7) gauges the child's emotional availability towards the parent (Biringen et al., 1998). It is indicative of his/her pleasure when interacting with the parent and how well the child responds to parental bids and expressions. Child Involvement (range 1-7) refers to the degree to which the child invites or includes the parent into play and expresses affect in this context. Regarding the child scales, lower points are applied to both 
non-optimal responses: under-responsive/under-involved and over-responsive/ over-involved (Biringen et al., 1998). All scales are ordinal, with higher scores representing higher quantity/quality of the characteristic involved.

All interactions were scored by three raters that had achieved reliability with the first author of the scales and that were blind to the other measures. Twenty-five cases ( $48 \%$ of the total sample) were double coded for inter-rater reliability. Good agreement was obtained for all scales using ICC: sensitivity (average ICC $=.88$ ), responsiveness to mother (average ICC $=.85$ ), and involvement of mother (average $\mathrm{ICC}=.80)$.

\section{Emotion regulation}

The Shape Sorter Task was specifically developed for this study. It was designed to assess a 10-month-old infant's style of emotion regulation during mother-infant interaction at home using a semi-structured problem solving task. The researcher gave a shape sorter to the mother and explicitly asked her to teach the infant how to insert the pieces in the holes. It took $10 \mathrm{~min}$ and was video-recorded for later scoring.

Because of the demands of a long-10 min-developmentally challenging game (it is recommended for 12-month-olds), infants are expected to express negative emotions, at some point, during the task. To the infant, the shape sorter is only an object to explore at will. Only the mother comprehends the task objective. Therefore, she will redirect the infant's attention and behaviours to the shape sorter even when the child is no longer interested in playing. These two aspects of the task make it likely to elicit negative emotions: while the duration of the task-10 min-increases the probability of the infants losing interest in it, it is expected that mothers will try their best to have the infants perform the task during the whole $10 \mathrm{~min}$. This is likely to create a conflict between infant and mother, thus predisposing for negative emotions to be activated in the infant.

The expression of emotion-or the absence thereof-is used to discriminate the quality of the emotion regulation style displayed by the infant. In a task that is long and difficult for the infant, over-regulation was identified when there was a total lack of expression of negative emotions by the infant throughout the duration of the task while the infant was highly focused on the shape sorter. The adaptive style of emotion regulation was characterized by the expression of some negative emotions that led to a momentary disruption of the task but that would be followed by the infant's shift to positive or neutral affect and renewed focus on the shape sorter. Finally, underregulation was used to label infants who expressed many negative emotions that disrupted the task; the infant was not successful in shifting the negative affect and refocusing on the shape sorter.

To assign each infant to one of the three styles of emotion regulation, an 8-point rating scale was developed: 8-no expression of negative emotionality; 7-rare expression of negative emotionality (Throughout the task, the infant shows rare signs of being distressed. These emerge towards the end of the interaction but are easily contained, and the infant refocuses on the task.); 5-some expression of negative emotionality (The infant becomes distressed at times; he/she is still able to resume some neutral or positive emotionality, but the disruption of the task is already substantial.); 3-frequent expression of negative emotionality (The infant spends most of the time displaying distress.); 1 - predominance of negative emotionality (Throughout the 10-min session, the infant is almost persistently distressed.). The remaining categories of 2,4, and 6 reflect situations in which the infants' behaviours throughout the session are placed between two adjacent categories. On the basis of 
this coding system, infants rated from 1 to 5 were further classified as displaying emotion under-regulation, infants rated from 6 to 7 were classified as displaying an adaptive style of emotion regulation, and infants rated 8 were classified as displaying emotion over-regulation.

After training with the first author, all interactions were independently scored by four trained judges. For reliability purposes, $67 \%$ of the videotapes were randomly selected and distributed to pairs of raters for double coding. We calculated the inter-rater reliability using the three categories (over-regulation, adaptive, and under-regulation), Cohen's $\kappa=.77, p<.001$.

\section{Infant temperament}

The Infant Characteristics Questionnaire (Bates et al., 1979) assesses the mother's perception of the infant's difficult temperament, globally defined as an infant who cries a lot and is difficult to soothe (Bates et al., 1979). Mothers were asked to rate the infant's behaviour on a 7-point scale from 1 (more optimal) to 7 (less optimal). We used the Portuguese translation (Pires, 1997) of the 6-month infant version (there is no 10-month version) as employed in previous investigations (see DeGangi, Porges, Sickel, \& Greenspan, 1993), and it includes 17 questions. The questionnaire has a good overall reliability, Cronbach's $\alpha=.75$ (Martins, Martins, \& Soares, 2006).

\section{Attachment quality}

The SSP (Ainsworth et al., 1978) was used to assess attachment quality at 12 or 16 months. The SSP is a standardized laboratory procedure that involves eight episodes designed to elicit mild to medium infant stress. A team of raters reliable in Ainsworth's A/B/C classification of attachments patterns and blind to all other measures assigned each child to one of the following categories: secure (B), insecure avoidant $(\mathrm{A})$, or insecure resistant $(\mathrm{C})$. Inter-rater reliability was adequate, Cohen's $\kappa=.93, p<.001$, achieved through double coding of 28 cases $(52.85 \%$ of the sample).

\section{RESULTS}

\section{Analytic Strategy}

We conducted a series of procedures aimed at selecting the predictors of emotion over-regulation. We tested the association of all styles of emotion regulation (overregulation, adaptive regulation, and under-regulation) with the three attachment patterns (insecure avoidant, secure and insecure ambivalent), using Cramer's V, as the expected cell count prevented us from using the chi-square test. This was followed by a series of analyses comparing two emotion regulation styles at a time using Bonferroni's correction to prevent Type I errors. In addition, we tested for emotion regulation group differences on the dyadic emotional interaction composite and temperament scales using ANOVAs with post hoc Gabriel tests. Finally, a multinomial logistic regression analysis was carried out to predict over-regulation (the reference category) versus adaptive and under-regulation.

\section{Descriptives and Preliminary Analyses}

Half of the infants $(n=26,50 \%)$ were classified into the category of adaptive emotion regulation, 11 infants (21.2\%) were classified into the over-regulation category, and 15 
$(28.8 \%)$ were classified into the under-regulation category. The attachment distribution was as follows: secure attachment $(n=36,69.2 \%)$ compared with $21.2 \%(n=11)$ insecure avoidant and $9.6 \%(n=5)$ insecure ambivalent/resistant. Descriptive statistics for the other variables can be found in Table 1.

Preliminary analyses were carried out to examine possible associations between study variables and sex or SES. No significant relations were found between sex and emotion regulation, $\chi^{2}(2)=0.74, p=.690$; attachment patterns, $\chi^{2}(2)=5.56$, $p=.062$; dyadic emotional interaction, $t(50)=-.36, p=.720$; and difficult temperament, $t(48)=-.43, p=.667$. Only difficult temperament was found to be associated with higher SES $\left(r_{s}=.33, p=.019\right)$. Mothers that perceived their infants as crying more and as being more difficult to soothe than other infants came from households with a higher SES. No relation was found between SES and emotion regulation, $\mathrm{K}-\mathrm{W}$ test, $\chi^{2}(2)=1.96, p=.375$; between SES and attachment patterns, $K-W$ test, $\chi^{2}(2)=2.27$, $p=.322$; nor between SES and dyadic emotional interaction, $r_{s}=-.02, p=.902$.

\section{Selecting Predictors of Over-regulation}

A medium-sized association between emotion regulation styles $(0=$ over-regulation, 1 = adaptive regulation, $2=$ under-regulation $)$ and attachment $(0=$ insecure avoidant, 1 = secure, 2 = insecure ambivalent) was found, Cramer's V=.34, $p=.020$. Overregulation versus under-regulation was found to be strongly associated with attachment, Cramer's V $=.57, p=.015$, whereas over-regulation versus adaptive regulation was not, Cramer's V =.42, $p=.041$, taking in consideration that we used the Bonferroni correction $(p<.017)$. Adaptive versus under-regulation was not found to be associated with attachment, Cramer's V =.21, $p=.408$ (see Table 1 for descriptives).

ANOVA revealed differences between emotion regulation styles, $F(2,49)=5.44$, $p=.007$, on the quality of the dyadic emotional interaction. Post hoc Gabriel tests showed that infants classified as over-regulatory had a lower quality of motherinfant emotional interaction than infants with an adaptive emotion regulation style $(p=.011)$. Additionally, no differences were found between over-regulation and under-regulation styles $(p=.78)$, and infants classified as having an adaptive emotion regulation had a tendency to have better mother-infant emotional interaction $(p=.068)$ than under-regulators.

No differences between the styles of emotion regulation were found for infant temperament, $F(2,47)=0.57, p=.569$.

Table 1. Attachment, dyadic emotional interaction and difficult temperament by emotion regulation styles $(N=52)$

Total Emotion regulation

$n$

\begin{tabular}{|c|c|c|c|c|}
\hline & & \\
\hline & & Over & Adaptive & Under \\
\hline Attachment, $n(\%)$ & & 11 & 26 & 15 \\
\hline Insecure avoidant & $11(21.20)$ & 6 & 4 & 1 \\
\hline Secure & $36(69.20)$ & 5 & 20 & 11 \\
\hline Insecure ambivalent & $5(9.60)$ & 0 & 2 & 3 \\
\hline Dyadic emotional interaction, $M(S D)$ & $15.37(3.02)$ & $13.55(1.97)$ & $16.15(2.43)$ & $14.33(2.72)$ \\
\hline Difficult temperament $^{\mathrm{a}} M(S D)$ & $43.58(7.55)$ & $41.90(8.91)$ & $43.32(7.92)$ & $45.13(6.02)$ \\
\hline
\end{tabular}

${ }^{\mathrm{a}} N=50$. 


\section{Predicting Over-regulation}

A multinomial regression analysis predicting emotion regulation styles-overregulation, under-regulation, and adaptive regulation-was conducted with overregulation as the reference category (Table 2). Dyadic emotional interaction and attachment were used as predictors given their association to emotion regulation. As expected in normative samples, the number of ambivalent infants $(n=5)$ was much lower than the other two attachment categories. To conduct the multinomial regression, we had to include these infants in one of the other categories. Because it was our hypothesis that avoidant attachment would predict emotion overregulation, we combined ambivalent attachment with secure attachment in a non-avoidant category. Thus, attachment quality was recoded into two groups $(0=$ avoidant attachment; $1=$ non-avoidant: secure or insecure ambivalent). For this variable, non-avoidant was used as the reference category.

The overall model fits the data well, $\chi^{2}(4)=18.56, p=.001$, and the Nagelkerke pseudo- $R^{2}$ was .34 . Both predictors have a significant impact on the model as ascertained by the likelihood ratio tests: dyadic emotional interaction, $\chi^{2}(2)=9.73, p=.008$; attachment patterns, $\chi^{2}(2)=8.14, p=.017$. Two sets of parameter estimates are provided in Table 2 (under-regulation versus over-regulation and adaptive emotion regulation versus over-regulation). By looking at the first set, we can conclude that the quality of dyadic emotional interaction does not emerge as a significant predictor. Therefore, the odds for an infant to be an overregulator or an under-regulator do not change as a function of differences in the quality of dyadic emotion interaction. Nevertheless, over-regulation is more likely than under-regulation if the infant has an avoidant attachment in comparison with a non-avoidant attachment (secure or insecure ambivalent). In the second set of parameter estimates, an over-regulation style is more likely to be identified than adaptive emotion regulation when there is lower quality of mother-infant emotional interaction. In addition, there is a trend (marginally significant result) for over-regulators to be identified if the infant's attachment pattern is avoidant in comparison with non-avoidant (secure or insecure ambivalent).

Table 2. Parameter estimates for multinomial logistic regression predicting emotion regulation styles

\begin{tabular}{lcccccc}
\hline & $B$ & $S E$ & Wald & $d f$ & $p$ & $\mathrm{e}^{B}$ \\
\hline Under-regulation versus over-regulation & -1.13 & 2.54 & 0.20 & 1 & .657 & \\
$\quad$ Intercept & 0.16 & 0.18 & 0.73 & 1 & .393 & 1.17 \\
Dyadic emotional interaction & -2.86 & 1.21 & 5.59 & 1 & .018 & 0.06 \\
Avoidant attachment & -5.72 & 2.83 & 4.08 & 1 & .043 & \\
Adaptive emotion regulation versus & over-regulation & & & & \\
Intercept & -5.48 & 0.20 & 6.14 & 1 & .013 & 1.62 \\
Dyadic emotional interaction & -1.67 & 0.89 & 3.55 & 1 & .060 & 0.19 \\
Avoidant Attachment & &
\end{tabular}

Note. For this analysis, $N=52: n=11$ for over-regulation, $n=26$ for adaptive regulation, and $n=15$ for under-regulation. Emotion over-regulation is the predictor reference category. Attachment coded as avoidant attachment or non-avoidant attachment (secure or insecure ambivalent): non-avoidant is the reference category. Positive $B$ values indicate that the emotion regulation style scored higher on the variable in question compared with the reference category (over-regulation). 


\section{DISCUSSION}

The main aim of this research was to contribute to the characterization of emotion over-regulation in infants by contrasting it with both emotion under-regulation (maladaptive) and an adaptive emotion regulation style. The results demonstrate that it is possible to differentiate over-regulation from the other two styles, sustaining the possibility of early identification of emotion over-regulation.

The first hypothesized association between avoidant attachment and emotion over-regulation has been documented. Previous studies yielded contradictory results, with some providing empirical validation for this connection in infancy (Braungart \& Stifter, 1991, Crugnola et al., 2011; Koulomzin et al., 2002), while others reporting null findings (Braungart-Rieker et al., 2001; NICHD Early Child Care Research Network, 2004; Volling et al., 2002). This investigation offers support in favour of the significance of the avoidant attachment relationship to the development of emotion over-regulation in infancy. First, avoidant attachment predicted over-regulation when compared with under-regulation. Second, we found a trend for avoidant attachment to predict over-regulation when compared with adaptive emotion regulation. Attachment theory suggests that avoidance may be conceived as a response to an attachment figure that is less warm (especially in situations of physical proximity), less responsive to emotional distress, and more intrusive, controlling, and overstimulating (Main, 1990). Main (1990) proposed that insecure avoidant infants develop a conditional attachment behavioural strategy in which the child inhibits the urge to seek close proximity with the attachment figure in situations of alarm. For this reason, the infant limits his/her emotional communication to the attachment figure while simultaneously developing other resources to cope with emotional arousal, such as diverting attention to the inanimate world of objects, constituting an emotional regulation style characterized by over-regulation (Cassidy, 1994; Sroufe, 2000).

The second hypothesis was also supported. This study furthered the knowledge of emotion over-regulation in infancy, as it demonstrated that lower quality of mother-infant emotional interactions predicted over-regulation (versus adaptive emotion regulation). This result is even more convincing given that dyadic emotional interaction was assessed in the home environment, a setting that may more easily represent behaviours found in everyday life. At the same time, the quality of dyadic emotional interaction did not differentiate over-regulation from under-regulation, with both being associated with lower scores compared with adaptive emotion regulation. Therefore, these results suggest that both styles, under- regulation, and over-regulation, may constitute maladaptive emotion regulation because research has demonstrated that less optimal mother-infant exchanges may hamper the development of emotion regulation (Calkins \& Hill, 2007; Schore, 2000; Sroufe, 1996). This study supports the hypothesis that this connection is also true for over-regulation.

This investigation strengthens the argument that avoidant attachment may have an important influence on the development of an emotion over-regulation strategy over and above the quality of mother-infant dyadic exchanges that are also emotionally relevant. This was our third hypothesis. When attachment and dyadic emotion interaction were tested together as predictors of emotion regulation in a multinomial logistic regression analysis, avoidant attachment was found to be a significant predictor of over-regulation when compared with under-regulation. Additionally, it was a marginally significant predictor when compared with adaptive emotion regulation. Looking closely at the different contrasts between the emotion regulation styles, it seems that mother-infant emotional interaction, or as we defined it, the capacity of both elements of the dyad-mother and infant-to communicate 
emotionally and become affectively attuned to each other on an everyday scenario (e.g., during play), is compromised in both over-regulation and under-regulation when compared with an adaptive style. However, when we analyse the relationship with regard to attachment, avoidant attachment specifically predicts over-regulation in comparison with adaptive and under-regulation styles. It seems that the repeated experiences that these infants go through when negative emotions arise and the attachment system is activated play an additionally important role in the development of emotion over-regulation. Contrary to some other investigations, the association between over-regulation and attachment cannot be accounted for by their overlapping empirical operationalization, as we assessed emotion regulation during a task in the infant's home rather than during the SSP. This approach, rarely adopted with infants (Diener et al., 2002), may also be more informative of the real life behaviour than laboratory assessments (Campos et al., 2011). The association found between avoidant attachment using the SSP and the emotion regulation style observed in the interaction with the mother in the infant's natural environment adds validity to the theoretically advocated relation.

This investigation also shows that attachment is one out of several factors related to the expression of the infant's emotion regulation style. Although avoidant attachment significantly predicted over-regulation when contrasted with under-regulation, only a marginal association between over-regulation and adaptive regulation was found. Our marginal results may support the necessity to consider other variables that contribute to a model of emotion regulation of infants. An examination of the relationship between temperament and attachment may be enlightening. Temperamental characteristics do not consistently explain individual differences in attachment (Vaughn, Bost, \& van IJzendoorn, 2008). Even so, some investigations have shown that temperamental reactivity and regulation do seem to influence the emotional characteristics presented by infants during the SSP. Negative emotionality may cut across attachment patterns so that avoidant babies are similar to B1 and B2 babies, while B4 are similar to ambivalent babies (Vaughn et al., 2008), such that temperament affects the type of security or insecurity but not the security or insecurity itself. In the same way, while attachment may influence the development of emotion regulation strategy, other variables, including individual emotional characteristics, may additionally underline the emotional regulation style. If this is the case, one might speculate that avoidant attachment emerged as a significant predictor because over-regulation and under-regulation are related to emotionality opposites (low versus high negative emotionality), while only a marginal association for the contrast between adaptive and over-regulation (categories closer in the levels of emotionality) emerged.

Nevertheless, with the temperament measures used in this study, there seems to be little support for the previous interpretation because no differences were found between over-regulation and the other styles, contradicting our fourth hypothesis. We hypothesized that over-regulated infants would be less emotionally difficult, following the suggestion of Eisenberg et al. (2007) that some temperament characteristics, namely rigid over-control in the expression of emotion, would be associated with emotion over-regulation. Some explanations for this result may be advanced. We used a temperament measure that relied on mothers' perceptions. Although it is a common methodology of assessment in the field of development (Rothbart \& Sheese, 2007), maternal perceptions of infant temperament may not accurately represent the biological contribution of the infant. It may instead reflect the history of care and/or parental bias as well as infant characteristics (MacKenzie \& McDonough, 2009). Additional research is needed to determine which temperamental characteristics may be associated with over-regulation. 
The results of this study attest to the validity of the new measure created to assess emotion regulation in infancy: Shape Sorter Task. Identifying overregulation is not easy, as infants that do not express negative emotions most often appear adaptive, which may, in part, explain the limited research available on the subject. In the development of this assessment procedure, we used specific indicators to identify emotion over-regulation (Cassidy, 1994; Keenan, 2000; Main, 1990; Sroufe, 1996). First, we noted the presence of over-regulation when infants did not express any negative emotions. It should be kept in mind that this task was designed to elicit negative emotions. It is long, increasing the likelihood of the infants losing interest in it, while it is expected that the mothers will try their best to have the infants perform the task during the whole $10 \mathrm{~min}$. Second, infants classified as over-regulators are highly focused on the task. We hypothesized that they would be playing with the shape sorter with a regulatory purpose, which is consistent with emotional over-regulation because focusing attention on objects or distracting toy play are behaviours that help inhibit negative affect (Graziano, Calkins, \& Keane, 2011; Kopp, 1989).

One of the limitations to this investigation is that we did not register psychophysiological correlates of infant emotion regulation during the Shape Sorter Task. Therefore, the validity of the three proposed styles of emotion regulation remains an open issue. Analysing relations to future child adaptive outcomes would also contribute to this end. Additionally, we did not control for the mother's behaviour in the Shape Sorter Task, which may have conditioned the scoring of this measure. Nevertheless, Diener et al. (2002) demonstrated that emotion regulation skills seem to become organized into patterns or styles starting as early as the end of the first year of life. This suggests that the infant is considerably influencing the final score on the Shape Sorter Task. If this is not the case, however, and it is the mother's behaviour that is greatly influencing the infant's emotional regulation style, there is a theoretical expectation that the infant will internalize these patterns and emotional regulation strategies (Sroufe, 1996). Therefore, the interaction during the Shape Sorter Task may be considered a reflection of the way the dyad has been resolving emotionally activating situations. On the basis of this framework, the results will be an index to the child's style of emotion regulation that he/she will use autonomously. Therefore, not controlling the mother's behaviour per se may not be as problematic as it may seem at face value.

Another important caveat to this study is that the design does not allow for a consubstantiation that attachment is causing the development of the emotion regulation styles and not vice versa, although the first causal direction is the one mainly supported in the literature (e.g., Braungart-Rieker et al., 2001; Crugnola et al., 2011).

Additionally, we used a global measure of the quality of mother-infant emotional interaction (dyadic emotional interaction). This assessment procedure does not allow for the identification of specificities of interactional exchanges related with over-regulation and under-regulation because of the format of the child's emotional availability scales used to create the dyadic emotional interaction composite. Lower points are both applied to under-responsive/ under-involved infants (low emotional communication with the parent) as well as to over-responsive/over-involved (the infant clings to the parent). Finally, because fathers have been identified as particularly important for the development of emotion regulation (Flanders et al., 2010), future research may expand current knowledge by analysing how father-infant relationships may impact upon the infant's emotion regulation in general and over-regulation specifically. 


\section{Conclusions and Clinical Implications}

This investigation offers an initial contribution towards the understanding and identification of emotion over-regulation in infancy. Most research on emotion regulation continues to operationalize the maladaptive style of emotion regulation as the high expression of negative emotionality (i.e., under-regulation), leaving the overregulation style unaddressed. We demonstrated that over-regulation (i) can be identified in infancy, and (ii) it is, in fact, a maladaptive style of emotion regulation associated with lower quality mother-infant interactional contexts (i.e., avoidant attachment and lower mother-infant emotional communication and attunement). Currently, there is agreement that emotion regulation processes have an impact on healthy versus maladaptive behaviour. They are part of the control processes (such as executive functioning) that allow children, adolescents, and adults to self-regulate, contributing to adaptive functioning across developmental domains and psychopathology (Calkins, 2010; Dillon et al., 2011). The results of this investigation highlight the importance of studying emotion over-regulation from an early age. Ultimately, new research may provide opportunities for early intervention and prevention of future maladaptive outcomes that arise from emotion over-regulation in infancy.

\section{ACKNOWLEDGEMENTS}

This research was supported by grants from the Portuguese Foundation for Science and Technology (SFRH/BD/16524/2004/254S) and from Neofarmacêutica. We thank all the colleagues that helped collect and code the data used for this article.

\section{REFERENCES}

Ainsworth, M., Blehar, M., Waters, E., \& Wall, S. (1978). Patterns of attachment: A psychological study of the strange situation. Hillsdale: Erlbaum.

Aldao, A., \& Nolen-Hoeksema, S. (2010). Specificity of cognitive emotion regulation strategies: A transdiagnostic examination. Behaviour Research and Therapy, 48, 974-983. DOI: 10.1016/ j.brat.2010.06.002

Bates, J. E., Freeland, C. A. B., \& Lounsbury, M. L. (1979). Measurement of infant difficultness. Child Development, 50, 794-803. DOI: 10.1111/1467-8624.ep7250994

Beebe, B., Jaffe, J., Markese, S., Buck, K., \& Chen, H. (2010). The origins of 12-month attachment: A microanalysis of 4-month mother-infant interaction. Attachment $\mathcal{E}$ Human Development, 12, 3-141. DOI: $10.1080 / 14616730903338985$

Biringen, Z., Damon, J., Grigg, W., Mone, J., Pipp-Siegel, S., Skillen, S., et al. (2005). Emotional availability: Differential predictions to infant attachment and kindergarten adjustment based on observation time and context. Infant Mental Health Journal, 26, 295-308. DOI: 10.1002/ imhj.20054

Biringen, Z., Robinson, J., \& Emde, R. N. (1998). The emotional availability scales (3rd ed.). Unpublished manual, Colorado State University.

Braungart, J. M., \& Stifter, C. A. (1991). Regulation of negative reactivity during the strange situation: Temperament and attachment in 12-month-old infants. Infant Behavior $\mathcal{E}$ Development, 14, 349-367. DOI:10.1016/0163-6383(91)90027-P

Braungart-Rieker, J. M., Garwood, M. M., Powers, B. P., \& Wang, X. (2001). Parental sensitivity, infant affect, and affect regulation: Predictors of later attachment. Child Development, 72, 252-270. DOI: 10.1111/1467-8624.00277

Calkins, S. D. (2010). Commentary: Conceptual and methodological challenges to the study of emotion regulation and psychopathology. Journal of Psychopathology and Behavioral Assessment, 32, 92-95. DOI: 10.1007/s10862-009-9169-6 
Calkins, S. D., \& Hill, A. (2007). Caregiver influences on emerging emotion regulation: Biological and behavioral transactions in early development. In J. Gross (Ed.), Handbook of emotion regulation (pp. 229-248). New York: Guilford Press.

Campos, J. J., Walle, E. A., Dahl, A., \& Main, A. (2011). Reconceptualizing emotion regulation. Emotion Review, 3, 26-35. DOI: 10.1177/1754073910380975

Cassidy, J. (1994). Emotion regulation: Influences of attachment relationships. Monographs of the Society for Research in Child Development, 59(2-3, Serial No. 240), 228-249.

Cicchetti, D., Ganiban, J., \& Barnett, D. (1991). Contributions from the study of high-risk populations to understanding the development of emotion regulation. In J. Garber, \& K. A. Dodge (Eds.), The development of emotion regulation and dysregulation (pp. 15-48). New York: Cambridge University Press.

Cole, P. M., Martin, S. E., \& Dennis, T. A. (2004). Emotion regulation as a scientific construct: Methodological challenges and directions for child development research. Child Development, 75, 317-333. DOI: 10.1111/j.1467-8624.2004.00673.x

Cole, P. M., Michel, M. K., \& Teti, L. O. (1994). The development of emotion regulation and dysregulation: A clinical perspective. Monographs of the Society for Research in Child Development, 59(2-3, Serial No. 240), 73-100.

Corriveau, K. H., Harris, P. L., Meins, E., Fernyhough, C., Arnott, B., Elliott, L., et al. (2009). Young children's trust in their mother's claims: Longitudinal links with attachment security in infancy. Child Development, 80, 750-761. DOI: 10.1111/j.1467-8624.2009.01295.x

Crugnola, C. R., Tambelli, R., \& Spinelli, M. (2011). Attachment patterns and emotion regulation strategies in the second year. Infant Behavior \& Development, 34, 136-151. DOI: 10.1016/j.infbeh.2010.11.002.

DeGangi, G. A., Porges, S. W., Sickel, R. Z., \& Greenspan, S. I. (1993). Four-year follow-up of a sample of regulatory disordered infants. Infant Mental Health Journal, 14, 330-343. DOI: 10.1002/1097-0355(199324)14:4<330::AID-IMHJ2280140407>3.0.CO;2-K

Diamond, L. M., \& Fagundes, C. P. (2008). Developmental perspectives on links between attachment and affect regulation over the lifespan. Advances in Child Development and Behavior, 36, 83-134. DOI: 10.1016/S0065-2407(08)00003-7

Diener, M. L., Mangelsdorf, S. C., McHale, J. H., \& Frosch, C. A. (2002). Infants' behavioral strategies for emotion regulation with fathers and mothers: Associations with emotional expressions and attachment quality. Infancy, 3, 153-174. DOI: 10.1207/S15327078IN0302_3

Dillon, D. G., Deveney, C. M., \& Pizzagalli, D. A. (2011). From basic processes to real-world problems: How research on emotion and emotion regulation can inform understanding of psychopathology, and vice versa. Emotion Review, 3, 74-82. DOI: 10.1177/1754073910380973

Eisenberg, N., Hofer, C., \& Vaughan, J. (2007). Effortful control and its socioemotional consequences. In J. J. Gross (Ed.), Handbook of emotion regulation (pp. 287-306). New York: Guilford Press.

Feldman, R., Greenbaum, C. W., \& Yirmiya, N. (1999). Mother-infant affect synchrony as an antecedent of the emergence of self-control. Developmental Psychology, 35, 223-231. DOI: 10.1037/0012-1649.35.1.223

Flanders, J. L., Simard, M., Paquette, D., Parent, S., Vitaro, F., Pihl R. O., \& Séguin J. R. (2010). Rough-and-tumble play and the development of physical aggression and emotion regulation: A five-year follow-up study. Journal of Family Violence, 25, 357-367. DOI: $10.1007 /$ s10896-009-9297-5

Goldberg, S., Grusec, J. E., \& Jenkins, J. M. (1999). Narrow view of attachment or broad view of protection? Rejoinder to the commentaries. Journal of Family Psychology, 13, 504-507. DOI: $10.1037 / 0893-3200.13 .4 .504$

Graffar, M. (1956). Une méthode de classification sociale d'echantillons de population [A method of social classification of samples of population]. Courrier, 6, 455-459.

Graziano, P. A., Calkins, S. D., \& Keane, S. P. (2011). Sustained attention development during the toddlerhood to preschool period: Associations with toddlers' emotion regulation strategies and maternal behaviour. Infant and Child Development. DOI: 10.1002/icd.731

Graziano, P. A., Keane, S. P., \& Calkins, S. D. (2010). Maternal behaviour and children's early emotion regulation skills differentially predict development of children's reactive control and later effortful control. Infant and Child Development, 19, 333-353. DOI: 10.1002/ICD.670 
Gross, J. J., \& Thompson, R. A. (2007). Emotion regulation: Conceptual foundations. In J. J. Gross (Ed.), Handbook of emotion regulation (pp. 3-24). New York: Guilford Press.

Keenan, K. (2000). Emotion dysregulation as a risk factor for child psychopathology. Clinical Psychology: Science and Practice, 7, 418-434. DOI: 10.1093/clipsy.7.4.418

Kopp, C. B. (1989). Regulation of distress and negative emotions: A developmental view. Developmental Psychology, 25, 343-354. DOI: 10.1037/0012-1649.25.3.343

Koulomzin, M., Beebe, B., Anderson, S., Jaffe, J., Feldstein, S., \& Crown, C. (2002). Infant gaze, head face and self-touch at 4 months differentiate secure vs. avoidant attachment at 1 year: A microanalytic approach. Attachment $\mathcal{E}$ Human Development, 4, 3-24. DOI: 10.1080/14616730210123120

MacKenzie, M. J., \& McDonough, S. C. (2009). Transactions between perception and reality: Maternal beliefs and infant regulatory behavior. In A. Sameroff (Ed.), The transactional model of development: How children and contexts shape each other (pp. 35-54). Washington, DC: American Psychological Association.

Main, M. (1990). Cross-cultural studies of attachment organization: Recent studies, changing methodologies, and the concept of conditional strategies. Human Development, 33, 48-61. DOI: $10.1159 / 000276502$

Martins, E. C., Martins, C., \& Soares, I. (2006, Outubro). Questionário de características do bebé: Adaptação à idade de 10 meses e resposta pelo pai e mãe [Infant characteristics questionnaire: Adaptation for 10 months with responses by mother and father]. Poster presented at XI Conferência Internacional Avaliação Psicológica: Formas e Contextos [XI International Conference of Psychological Assessment: Ways and Context], Braga, Portugal.

Maughan, A., \& Cicchetti, D. (2002). Impact of child maltreatment and interadult violence on children's emotion regulation abilities and socioemotional adjustment. Child Development, 73, 1525-1542. DOI: 10.1111/1467-8624.00488

Meins, E., Fernyhough, C., Arnott, B., Vittorini, L., Turner, M., Leekam, S. R., et al. (2011). Individual differences in infants' joint attention behaviors with mother and a new social partner. Infancy, 16, 587-610. DOI: 10.1111/j.1532-7078.2010.00065.x

Mikulincer, M., \& Shaver, P. R. (2008). Adult attachment and affect regulation. In J. Cassidy, \& P. R. Shaver (Eds.), Handbook of attachment: Theory, research, and clinical applications (2nd ed., pp. 503-531). New York: Guilford.

Miller, A. L., McDonough, S. C., Rosenblum, K. R., \& Sameroff, A. J. (2002). Emotion regulation in context: Situational effects on infant and caregiver behavior. Infancy, 3, 403-433. DOI: 10.1207/S15327078IN0304_01

Morris, A. S., Silk, J. S., Steinberg, L., Myers, S. S., \& Robinson, L. R. (2007). The role of the family context in the development of emotion regulation. Social Development, 16, 361-388. DOI: $10.1111 / j .1467-9507.2007 .00389 . x$

Newland, R. P., \& Crnic, K. A. (2011). Mother-child affect and emotion socialization processes across the late preschool period: Predictions of emerging behaviour problems. Infant and Child Development, 20, 371-388. DOI: 10.1002/icd.729

NICHD Early Child Care Research Network. (2004). Affect dysregulation in the motherchild relationship in the toddler years: Antecedents and consequences. Development and Psychopathology, 16, 43-68. DOI: 10.1017/S0954579404044402

Pires, A. (1997). A percepção que a mãe tem do temperamento do bebé: Desenvolvimento da percepção e a sua relação com o comportamento da criança e com o comportamento, irritabilidade e auto-eficácia maternas [Mother's perception about her baby: Perception's development and its relation with the baby's behavior and mother's behavior, irritability and self-efficacy]. Unpublished doctoral thesis, Faculdade de Psicologia e de Ciências da Educação da Universidade do Porto, Porto.

Rothbart, M. K., \& Sheese, B. (2007). Temperament and emotion regulation. In J. J. Gross (Ed.), Handbook of emotion regulation (pp. 331-350). New York: Guilford Press.

Schore, A. N. (2000). Attachment and the regulation of the right brain. Attachment $\mathcal{E}$ Human Development, 2, 23-47. DOI: 10.1080/146167300361309

Srivastava, S., Tamir, M., McGonigal, K. M., John, O. P., \& Gross, J. J. (2009). The social costs of emotional suppression: A prospective study of the transition to college. Journal of Personality and Social Psychology, 96, 883-897. DOI: 10.1037/a0014755 
Sroufe, L. A. (1996). Emotional development: The organization of emotional life in the early years. New York: Cambridge University Press.

Sroufe, L. A. (2000). Early relationships and the development of children. Infant Mental Health Journal, 21, 67-74. DOI: 10.1002/(SICI)1097-0355(200001/04)21:1/2<67::AID-IMHJ8>3.0. $\mathrm{CO} ; 2-$

Thompson, R. A. (2008). Measure twice, cut once: Attachment theory and the NICHD study of early child care and youth development. Attachment $\mathcal{E}$ Human Development, 10, 287-297. DOI: $10.1080 / 14616730802113604$

Vaughn, B. E., Bost, K. K., van IJzendoorn, M. H. (2008). Attachment and temperament: Additive and interactive influences on behavior, affect, and cognition during infancy and childhood. In J. Cassidy \& P. R. Shaver (Eds.), Handbook of attachment: Theory, research, and clinical applications (2nd ed., pp. 192-216). New York: Guilford Press.

Volling, B. L., McElwain, N. L., Notaro, P. C., \& Herrera, C. (2002). Parents' emotional availability and infant emotional competence: Predictors of parent-infant attachment and emerging self-regulation. Journal of Family Psychology, 16, 447-465. DOI: 10.1037/ /0893-3200.16.4.447 